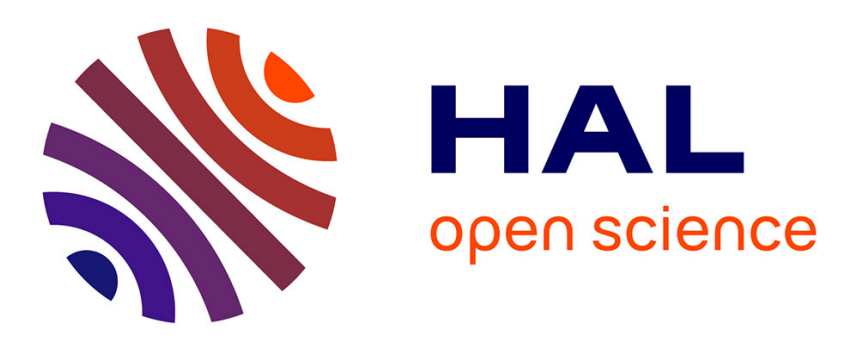

\title{
De la dépendance à l'institutionnalisation des soins de longue durée dans le grand vieillissement : perspectives psychanalytiques autour des dynamiques du passage
} Celine Racin

\section{- To cite this version:}

Celine Racin. De la dépendance à l'institutionnalisation des soins de longue durée dans le grand vieillissement: perspectives psychanalytiques autour des dynamiques du passage. Revue de psychothérapie Psychanalytique de Groupes, 2019, Clinique des réseaux, 73, pp.199-210. 10.3917/rppg.073.0199 . hal-03017542

\author{
HAL Id: hal-03017542 \\ https://hal.science/hal-03017542
}

Submitted on 20 Nov 2020

HAL is a multi-disciplinary open access archive for the deposit and dissemination of scientific research documents, whether they are published or not. The documents may come from teaching and research institutions in France or abroad, or from public or private research centers.
L'archive ouverte pluridisciplinaire HAL, est destinée au dépôt et à la diffusion de documents scientifiques de niveau recherche, publiés ou non, émanant des établissements d'enseignement et de recherche français ou étrangers, des laboratoires publics ou privés. 


\title{
DE LA DÉPENDANCE À L'INSTITUTIONNALISATION DES SOINS DE LONGUE DURÉE DANS LE GRAND VIEILLISSEMENT : PERSPECTIVES PSYCHANALYTIQUES AUTOUR DES DYNAMIQUES DU PASSAGE
}

\author{
CÉLINE RACIN
}

\begin{abstract}
«Le monde n'est qu'une branloire pérenne. Toutes choses y branlent sans cesse [...] La constance même n'est autre chose qu'un branle plus languissant. Je ne puis assurer mon objet. Il va trouble et chancelant, d'une ivresse naturelle. Je le prends en ce point, comme il est, en l'instant que je m'amuse à lui. Je ne peins pas l'être. Je peins le passage. »

Montaigne, Euvres complètes, 1962, Livre iii
\end{abstract}

Tout au long de la vie, il existe des lieux que les transitions biographiques convoquent, qui augurent des risques, des difficultés potentielles quant à leur traversée, des passerelles par lesquelles le passage devient initiation pour des expériences impliquant des franchissements de seuils définis comme " épreuves ». Dans la mesure où l'hospitalisation en service de gériatrie catalyse un certain nombre des enjeux psychiques liés à l'institutionnalisation des soins au cours du vieillissement, le franchissement du seuil hospitalier signe métaphoriquement la confrontation à la complexité du vieillir et à la diversité de ses formes, dont certaines offrent le miroir ambigu d'un devenir placé sous le sceau de la dépendance. L'hospitalisation, lorsqu'elle ouvre sur la nécessité de recourir à une institutionnalisation des soins de longue durée, que ce soit sous la forme d'un déménagement en établissement d'hébergement (ehpad) ou sous la forme de l'introduction durable et majeure d'aides professionnelles sur le lieu du domicile habituel, s'apparente notamment à une épreuve de réalité qui défait le sujet de certains des étayages antérieurs et le confronte douloureusement à la finitude de son être. Se trouve dès lors posée la question de la disposition au changement,

Céline Racin, Maître de conférences en psychologie clinique et psychopathologie, Université de Strasbourg - Faculté de Psychologie, Laboratoire Subjectivité, lien social et modernité (ea 3071 - suıisom) ; Psychologue clinicienne, hôpital Sainte-Périne (ap-hp) ; racin@unistra.fr 
infléchie par l'entrecroisement des causalités interne et externe, sans laquelle tout franchissement de seuil se verrait refusé.

retour sur 1'institutionnalisation : du Cadre au proCessus

Le terme d'institutionnalisation, fortement saturé de représentations multiples et ambivalentes, s'est aujourd'hui fermement installé dans le corpus lexical qui accompagne, soutient et encadre les directives et pratiques relatives à la prise en compte de la dépendance. Dans ce contexte, on peut s'étonner que ce terme fasse si rarement l'objet d'une entreprise de spécification, comme s'il allait de soi, bien qu'il soit utilisé avec des significations assez différentes selon le contexte où il apparaît. On trouvera ainsi employée en divers lieux l'expression institutionnalisation de la vieillesse, dans la continuité de l'institutionnalisation du cours de la vie ${ }^{1}$ (Kohli, 1986), lorsqu'il s'agit de souligner le traitement particulier accordé à une catégorie de population, qui consacre la convergence entre âge chronologique et rôles sociaux. On rencontrera ailleurs la question de l'institutionnalisation de la dépendance (Thomas, 2005), qui dénonce par exemple l'aménagement systématique de règles, de cadres et d'outils qui normalisent des aspects de l'expérience et de la prise en charge de la dépendance au moyen d'un paradigme privilégié, notamment biomédical (Frinault, 2005). Mais trop souvent encore, dans le langage courant, l'emploi isolé du terme d'institutionnalisation s'applique de manière implicite aux individus eux-mêmes, c'est-à-dire aux hommes et aux femmes qui sont concernés par l'expérience de la dépendance, et désigne le moment, l'action ou le processus par lequel une personne âgée s'installe en établissement d'hébergement à défaut de pouvoir se maintenir à son domicile, ce qui contribue à connoter très négativement cette expérience. Cette incertitude quant au terme et à ses divers emplois est rarement levée lorsqu'il s'agit de qualifier les situations dans lesquelles les sujets âgés, leurs proches et les soignants se trouvent engagés, avec le risque que cette incertitude - comme nous met en garde L. Boltanski (2008) -, d'abord sémantique, ne devienne aussi déontique, à propos de ce qui est, de ce qui importe, de ce qui a valeur, de ce qu'il convient ou non de respecter. Aussi, accordons-nous une attention toute particulière à préciser l'usage que nous faisons de la notion d'institutionnalisation rapportée au contexte de dépendance dans le grand vieillissement, en faisant notamment travailler les apports de la psychanalyse.

Sous la plume de C. Castoriadis (1975), le processus d'institutionnalisation relève d'un double mouvement qui requiert qu'une dynamique instituante (composante imaginaire qui est puissance de création, de production et d'invention de sens) parvienne à se fixer, à se stabiliser en des formes instituées (composante fonctionnelle qui

1. Cette perspective se trouve notamment nuancée par les travaux d'A.-M. Guillemard (2010). 
représente la sédimentation en un réseau symbolique de données, de significations culturelles et historiques préalablement articulées). Cette dynamique processuelle définit ainsi l'institution comme toujours faite, mais également toujours en train de se faire, de sorte qu'elle ne peut être appréhendée de manière statique. Ainsi, alors que le mouvement instituant vise à proposer des normes, conventions et lois aménageant de nouveaux rapports sociaux adaptés à l'institution actuelle, le mouvement d'établissement et de consolidation de l'institué permet qu'un certain degré de permanence puisse être trouvé pendant un temps. La dynamique instituant/institué, par le mouvement même d'autolimitation qui l'anime, permet d'assurer à l'institution l'ancrage nécessaire à sa localisation et à son ouverture au monde.

Ajoutons par ailleurs que, pour que l'institution soit investie - et donc utilisée, notamment aux fins du processus qu'elle convoite -, il faut nécessairement qu'une relation de congruence, d'adéquation ou d'homologie existe entre l'objet-institution créé par le sujet ou les groupes, et l'objet-institution trouvé dans l'environnement. De ce fait, la question de l'institutionnalisation rencontre la réflexion portant sur les conditions qui permettent d'accueillir, de rendre intelligible et d'accompagner un processus d'appropriation subjective de ce qui est donné à vivre. Dans la situation qui nous occupe, il s'agit de favoriser la mise au travail des problématiques singulières engagées dans le vécu de la dépendance, qui ne peuvent se comprendre que dans la transaction, la négociation que le sujet âgé a avec son propre vieillissement. Les psychanalystes qui ont nourri l'élaboration d'une véritable métapsychologie du cadre psychanalytique et des dispositifs thérapeutiques sont, à cet endroit, d'un précieux secours pour penser les aménagements privilégiés pour délimiter un rapport au temps et à l'espace apte à favoriser et soutenir le travail psychique. J. Bleger propose ainsi qu' " une relation humaine qui dure des années, au sein de laquelle se maintient ${ }^{2}$ un ensemble de normes et d'attitudes n'est rien moins qu'une véritable définition de l'institution. Le cadre est donc une institution à l'intérieur des limites de laquelle se produisent certains phénomènes auxquels nous donnons le nom de comportement ${ }^{3} \gg$. Tout l'enjeu est ensuite de spécifier à quelles conditions un ensemble de normes et de pratiques est-il susceptible d'être stabilisé, de se maintenir. Dans ce qu'il nomme la situation psychanalytique, l'auteur distingue un non-processus qui renvoie au cadre entendu comme un ensemble de constantes, et un processus évoluant et variant à l'intérieur du cadre et des limites du dispositif, qui constitue l'objet d'observation, d'analyse et d'interprétation. Derrière la question des conditions, c'est bien celle des dispositifs à travers lequel s'incarne le cadre thérapeutique, qui se trouve ici posée,

2. C'est nous qui soulignons.

3. J. Bleger, « Psychanalyse du cadre psychanalytique » (1966), dans R. Kaës (sous la direction de),

Crise, rupture et dépassement, Paris, Dunod, 2004, p. 259. 
non réductible à sa dimension opératoire. J.C. Rouchy souligne ainsi combien « il ne s'agit pas de programme, de protocole ou de procédures où l'ordre et la succession d'opérations seraient déterminés à l'avance. C'est la construction d'un espace dans lequel ce qui se passe et se dit prend sens par le fait même que cela se produit ou se reproduit dans cet espace, donnant comme le dit S. Resnik (1989) une "visibilité" aux processus inconscients ${ }^{4} \gg$. Avec D. Mellier, nous retenons par ailleurs la valeur fonctionnelle du dispositif, qui constitue « une "préparation" pour un travail arrêté comme possible. [...] Un dispositif suppose une "disposition", un état d'esprit, une manière d'être ou "une tendance à" ${ }^{5} »$.

Il reste évidemment à spécifier le type de " travail » (autrement dit le type de processus) qui est recherché par l'utilisation de tel ou tel dispositif et, à cette fin, à expliciter la théorie qui s'actualise dans tel ou tel dispositif dans le but de réunir les conditions de possibilités requises pour ce travail. Pour peu que les individus, les groupes de professionnels et les institutions accordent ensemble une valeur positive à la réalisation possible - même si elle n'est jamais totalement accomplie de ce travail, un autre regard peut dès lors être porté sur le processus d'institutionnalisation. Tâche sisyphéenne que celle-ci cependant, dans un contexte pétri de "normalisation des soins " directement consécutive à l'argument de réalisme économique ambiant (Dejours, 2016), qui attaque et menace fortement les embasements même de cette élaboration. Face au malaise dans les soins qui s'exprime dans nombre d'établissements gériatriques, il y a là un enjeu de taille à résister et à soutenir l'attention portée aux dispositions psychiques et matérielles qui participent des conditions de possibilité de ce travail. Notre propos s'inscrit dans cette perspective.

de 1'institutionnalisation des dispositifs de soins de longue durée

En nous appuyant sur les travaux de R. Roussillon (2012), qui a activement œuvré à une théorisation des dispositifs thérapeutiques, il apparaît déjà que penser l'institutionnalisation dans la situation qui nous occupe, c'est penser le processus de stabilisation des conditions de rencontre entre un sujet âgé confronté à la réduction des moyens de réalisations des activités nécessaires à la vie, et un sujet disposé à l'aider, à prendre soin de lui. Cette exigence requiert, dans notre perspective, de penser les conditions de rencontre rendant possible le travail de care, ce travail qui renvoie simultanément à un ensemble de dispositions psychiques et d'activités permettant de soutenir la vie (Tronto, 1993). En fonction des particularités des problématiques et

4. J.C. Rouchy, « La conception du dispositif de groupe dans différents cadres institutionnels », Revue de psychothérapie psychanalytique de groupe, $\mathrm{n}^{\circ} 47,2006$, p. 10.

5. D. Mellier, « Précarité psychique et dispositifs d'intervention clinique » (2006), Pratiques psychologiques, $\mathrm{n}^{\circ} 12$, p. 150. 
des tableaux cliniques de dépendance, plusieurs types de dispositifs ont été inventés pour étayer les différents aspects du travail de soin en situation de dépendance, et favoriser l'adéquation la plus heureuse possible entre la problématique centrale des sujets et le confort de travail des « répondants » potentiels que sont les professionnels du soin et de l'aide. Il existe ainsi différents dispositifs de soins qui structurent une situation dans laquelle le travail de care devient possible, c'està-dire dégagé de certaines contraintes de la réalité, notamment matérielles et biologiques, qui viendraient menacer la stabilité du lien, et dans laquelle le travail de care est rendu utilisable pour celui ou celle qui y fait appel (l'appel n'étant pas, dans notre propos, l'équivalent d'une demande formulée et adressée). S'il existe donc bien différentes façons de s'intéresser à la question de l'institutionnalisation, nous souhaitons, de notre côté, exploiter cette acception qui permet de faire porter l'accent de ce processus de stabilisation sur des pratiques et des normes et, au regard de notre propos, sur des pratiques et des normes particulières référées au domaine du soin. Cette conceptualisation permet d'emblée de s'extraire de la réduction dont procède la fameuse, et inopportune, formulation " institutionnalisation des personnes âgées ", qui court le risque d'assigner le sujet à une position passive, de le réifier, d'en faire le spectateur muet et immobile d'un processus sur lequel il n'aurait en définitive aucune prise, d'un processus par rapport auquel il serait incapable d'entretenir un rapport subjectif et d'investir une activité réflexive. La stabilisation de ces conditions de rencontre, au cours du vieillissement, procède de la mise en place et du recours à des dispositifs de soins dits " de longue durée " en France, encore dénommés «long term care support or services » dans les pays anglosaxons. Les systèmes de relations, de normes et de pratiques structurés par ces arrangements stabilisés (ces «non-processus ») prennent dans notre société des formes contrastées, dont les plus fréquentes sont les dispositifs de " soins à domicile " (in-home support) et les dispositifs abrités au sein d'établissements d'hébergement pour personnes âgées dépendantes (nursing home).

Au-delà des caractéristiques manifestes de ces dispositifs, c'est surtout l'investissement que fait le sujet âgé du projet d'institutionnalisation, qui participe hautement à configurer une topographie singulière, subjective, de cette rive du passage vers laquelle jeter l'ancre, ainsi qu'à dessiner différentes figures de passeurs rencontrés à cet endroit. Très souvent, la stabilité des liens visée par ces dispositifs, censée être porteuse de continuité et de sécurité, ne relève pas d'un acquis, et doit être progressivement conquise pour que le «non-processus » puisse s'instaurer comme tel. À cet égard, du fait de l'initiation qu'elle propose à une relation de soin confiée à des professionnels, l'hospitalisation en service de gériatrie constitue déjà une expérience inaugurale, une première rencontre avec les enjeux de l'institutionnalisation. La qualité des rencontres et du processus clinique qui s'y 
déploient s'avère bien souvent un révélateur de l'investissement d'une relation de soin et d'une certaine tolérance à l'intervention d'un autre pour soi, dont dépend en partie la préparation ou l'impréparation aux transformations sur lesquelles ouvre l'hospitalisation, sculptées par le recours durable à une institutionnalisation. Aussi ce passage exige-t-il de la part des professionnels de porter une attention soutenue au travail psychique susceptible de se mobiliser sur cette scène hospitalière. En effet, l'hospitalisation ne fait pas qu'accueillir ce passage, elle vient aussi l'encadrer en proposant ses modes propres de mise en sens, ses propres supports de figuration des transformations que le sujet traverse.

du rite de passage au passage comme rite :

fonctions psyChiques des ritualités institutionnelles

Dans nos sociétés qui accompagnent encore les mises en forme du naître et du devenir adulte, force est de constater que nous ritualisons peu le passage aux âges tardifs ${ }^{6}$. Dans ces conditions, le passage par l'hospitalisation, pour matériel qu'il soit le plus souvent, constitue aussi un attracteur possible d'un travail de symbolisation par lequel les sujets vieillissants s'approprient l'expérience vécue, tentent d'y donner un sens, là où les ritualités modernes font défaut, là où les " points de certitude symboliques » (Aulagnier, 1975) viennent à manquer et nécessitent d'être réinstitués par les sujets eux-mêmes. À l'hôpital, la structuration spatiale (sculptée notamment par l'écart entre les particularités du chez-soi et la configuration particulière, impersonnelle et hygiéniste, de la chambre d'hôpital) et temporelle (suspendue aux séquences ordonnées des soins et à la durée de l'hospitalisation) de la prise en charge du changement, permet en même temps d'en border les contours, de délimiter ses effets, d'offrir un point de butée qui limite le libre écoulement des excitations et s'offre comme appui au travail psychique. Plus encore, à l'hôpital, la relation d'objet est prégnante, particulièrement investie autour des soins qui constituent le ciment des liens. Le sujet âgé y fait l'expérience parfois nouvelle, inédite, d'être l'objet d'un travail de care, ce fameux travail qui désigne " des activités spécialisées où le souci des autres est explicitement au centre » (Molinier, 2010), le travail des infirmier-e-s et des aides-soignant-e-s en représentant une facette exemplaire. Les réponses à la fragilité d'autrui, caractéristiques de ce travail, utilisent des modes d'expression extrêmement variés, situés sur un continuum évoluant du pôle des savoir-faire les plus codifiés par les protocoles hospitaliers, au pôle mobilisant l'improvisation quotidienne et l'invention spontanée, en passant par l'entre-deux du « projet de vie » individualisé (Noël, 2012).

6. Contrairement aux festivités qui accompagnent certaines transitions vers de nouvelles étapes de la vie individuelle et sociale, à l'instar du « pot de départ à la retraite », qui demeure une tradition fort respectée. 
Les activités de soin, procurées au plus près des fonctions du corps et de la satisfaction des besoins vitaux, mais également les " effets de présence »(Puget, 2005), constituent les vecteurs privilégiés de ces réponses. Pour le sujet âgé qui rencontre des limitations nouvelles dans son indépendance fonctionnelle, les pratiques soignantes qui organisent les moments du lever, de la toilette, de l'habillage, du repas, du coucher, etc. peuvent avoir l'effectivité d'un rite de passage, entendu comme un « ensemble de conduites individuelles ou collectives, relativement codifiées, ayant un support corporel (verbal, gestuel, postural), à caractère plus ou moins répétitif, à forte charge symbolique ${ }^{7} \gg$.

Ainsi, si l'institution hospitalière n'a pas cette intention a priori de proposer des rites qui ordonnent le social à partir d'un mythe de référence (sauf à considérer le paradigme biomédical comme un mythe moderne), il apparaît que l'espace-temps hospitalier aménage une " gestation de l'entre-deux » qui propose une narration, une dramatisation non sue comme telle, mais qui a valeur de signifiant et qui permet à la temporalité psychique d'être soutenue par une temporalité explicite, visible, douloureuse, imputable au réel. Il nous semble que les ritualités institutionnelles proposent notamment une mise en scène de la vulnérabilité corporelle et du mystère de la mort, qui soutient le travail psychique de renoncement aux fantasmes d'indépendance qui préservaient jusque-là la représentation d'un corps intègre, complet, ferme, performant, résistant et éternel, pour apprivoiser un corps mortel, vulnérable, pénétrable, soumis aux affres du temps qui passe. Elles organisent un espace de séparation et de médiation qui permet au sujet de se confronter à ses peurs, notamment à la peur de la mort solitaire, qui répond au modèle de la mort hospitalière s'imposant avec l'évolution des progrès médicaux.

Il nous semble ainsi qu'il existe, à l'hôpital, des « rituels » qui marquent le corps de stigmates, ou, plus justement, des " pratiques » $\mathrm{du}$ corps plus ou moins codifiées par le travail de care, qui sont susceptibles d'être interprétées comme stigmates et constituer des opérateurs de l'organisation temporelle d'un avant, dont le sujet est séparé, et d'un après, marqué par le partage de son territoire intime dans les pratiques de care, témoignant du passage, du franchissement d'un seuil, d'un changement parfois irréversible, de l'avancée, dans tous les cas, dans la vieillesse. Ces pratiques s'appuient tant sur des processus archaïques (à travers la manière de se sentir accueilli, porté, nourri, bercé, caressé, contenu par les voix et les rythmes, la tenue et la manipulation du corps véhiculant des sensations de chaud, froid, doux, dur, rapide, lent, fluide, heurté...) que sur des processus secondaires placés sous le signe du registre symbolique. Face à la situation de crise à laquelle répondent l'offre d'hospitalisation et la perspective d'une institutionnalisation pour un sujet âgé, les pratiques soignantes

7. C. Rivière, Les rites profanes, Paris, Puf, 1995, p. 11. 
constituent autant de ritualités institutionnelles permettant à la psyché, en quête de sens, de trouver dans la scène hospitalière des supports de figuration pour apprivoiser les pertes et les séparations nécessaires. Par leur aspect répétitif et prévisible, ces rituels remplissent également une fonction sécurisante face à la menace du changement, à l'incertitude de la nouveauté, à la précarité ontologique, en proposant une temporalité qui défait le flottement dans lequel s'engouffre l'événement déstabilisateur. L'enjeu de ces pratiques, en service de gériatrie, nous semble recouvrir un double aspect : il s'agit à la fois de prévenir les dangers qui guettent le sujet âgé fragilisé par les percussions lourdes des coups du corps (et, par la même occasion, de contenir l'angoisse des proches quant à sa vulnérabilité) et de maintenir et favoriser des capacités fonctionnelles lui permettant d'exercer un pouvoir d'action et une maîtrise aussi étendue que possible sur les choses qui le concernent. Ce dernier objectif n'est pas sans reposer sur une certaine ambivalence, tant, dans l'ombre du soutien à l'indépendance fonctionnelle (physique et cognitive) de l'individu, se tient aussi tout l'enjeu du maintien de son appartenance comme sujet à la communauté des humains, appartenance dont le principe organisateur repose notamment sur la valeur ambiguë d'autonomie.

La fonction de liaison assurée par ces rituels de care tient notamment à leur caractère répétitif, qui n'a de valeur structurante et intégratrice que dans l'ouverture à la mêmeté plutôt que dans la fixation à l'identique, et donc dans le degré de liberté, d'improvisation et de spontanéité que les pratiques soignantes autorisent. Car les rituels de care ne sont pas seulement contraints par la nécessité biologique, mais puisent éminemment dans les sources pulsionnelles de la dépendance, ce qui explique la multitude des façons de faire, d'intervenir pour procurer l'action spécifique. C'est également ce qui confère à ces pratiques un "plus de care » par lequel le soignant accepte d'occuper une position de « témoin » de l'état interne du sujet, qui cherche à maîtriser ce qui le menace dans la dépendance, et qui n'a pas véritablement de nom, ce quelque chose qui échappera toujours quelque part à ce qu'il peut identifier, et qui dès lors risque de déborder et d'excéder les capacités d'élaboration psychique individuelle et groupale. L'opportunité, pour ces rituels, de s'offrir comme lieu de figuration et de support à l'élaboration, repose moins sur le perpétuel recommencement des opérations - dans leur temporalité (rythmicité quotidienne) et dans leur forme (similitude des séquences gestuelles) - que sur la possibilité de reprise à laquelle ils ouvrent, sur leur capacité à se départir d'une reproduction immuable pour intégrer la nouveauté et l'invention dans la répétition, pour tenir ensemble passé et présent, mouvements régrédient et progrédient. Cette caractéristique n'est cependant pas suffisante pour faire de ces rituels le lieu de toutes les potentialités, et faire qu'une fonction symbolisante et réflexive puisse s'y déployer. En effet, tout en puisant dans les satisfactions et déplaisirs propres 
aux expériences psychiques et affectives, plus ou moins avouables, vécus en situation de dépendance, ces pratiques ont pour grand intérêt de chercher en même temps à retraduire ces expériences, avec plus ou moins de fluidité ou de heurt, pour être réappropriées par le sujet. Cette opération se réalise dans un échange entre les registres du réel et de l'imaginaire, au regard duquel l'absence de jugement d'existence, de décision quant à l'appartenance de la dépendance à l'un ou l'autre monde, permet de maintenir le champ de l'illusion. Ce champ de l'illusion favorise l'introjection d'éléments nouveaux, fournis par les pratiques de care, dans les anciennes habitudes du sujet relatives aux soins du corps, à condition que le nouveau ne se substitue pas au passé mais que le passé subsiste sous une nouvelle forme dans le présent. Cette caractéristique, en ce sens, offre aussi les conditions d'un aprèscoup, qui donne la possibilité au sujet de réinterpréter les événements antérieurs, de jeter un nouvel éclairage sur les difficultés parfois tapies dans l'ombre dans le déroulement continu du quotidien au domicile, et de rendre représentable et partageable le vécu de dépendance. C'est ainsi que l'expérience de séparation d'avec le monde antérieur et la recherche de contenants que fait vivre l'hospitalisation peut raviver, dans l'après-coup, les représentations du domicile comme d'un ventre symbolique, lieu de sécurité et de protection, en contrepoint de ce qui vient cruellement à manquer, la maison devenant le lieu de projection du manque. Ainsi, le rapport au chez-soi, tel qu'il s'exprime au cours d'une hospitalisation, ne dit pas seulement quelque chose de l'investissement de cet objet, mais également du travail psychique en cours.

Définir les ritualités institutionnelles de la scène hospitalière comme rite de passage n'est pas sans rencontrer cependant une limite interprétative cruciale. En effet, l'institutionnalisation des soins ne représente pas un passage obligé au cours du vieillissement : contrairement aux rituels de passage traditionnels des sociétés prémodernes, l'institutionnalisation fait exister une préséance du besoin sur le sens et ne concentre pas des seuils que la culture incombe de franchir. En ce sens, nous nous intéressons moins à l'institutionnalisation des soins comme à un rite de passage, que comme un rite $d u$ passage, ou au passage comme rite, offrant une initiation au sujet âgé qui lui permet d'être conduit vers un certain nombre de transformations en fonction des paliers d'aménagements, plus ou moins dégageants, remis sur le métier par le travail psychique potentiellement mobilisé par la traversée du vieillissement (Verdon, 2013).

institutionnalisation : entre « effets de sens 》 et 《 effets d'assignation 》

Si une fonction essentielle du rite est de favoriser un travail de liaison en proposant des formes à des questions énigmatiques, il reste également à déterminer à quoi l'institutionnalisation des soins à l'hôpital propose un discours, une figuration. Il nous semble notamment que 
l'hospitalisation vient scander un passage, celui du temps, irréductibilité qui trouve un écho métapsychologique dans le travail du vieillir, et proposer une figuration sur l'origine, le temps qui passe, et la mort, fortement adossée à l'épistémè médicale. Le sens ainsi proposé à travers les activités soignantes qui sont subordonnées au discours et aux pratiques médicales à l'hôpital, est celui qui suppose que le vieillir arrive par le biologique, par le corporel. Dans l'ensemble, ces activités organisées autour de la précarité biologique ont ainsi pour fonction de contenir les problématiques psychiques liées à la dépendance, articulées aux angoisses de perte, de passivité, et finalement de mort, auxquelles confronte le temps qui passe et dont le corps du vieillard (r)éveille la perception. Les efforts déployés pour maintenir l'échéance du terme la plus éloignée possible revêtent une dimension paradoxale : tout en exhibant les corps à l'excès, les pratiques soignantes participent en même temps à l'occultation des signes de dégénérescence qui entretiennent une inquiétante proximité avec la mort, qu'il s'agisse de la mort physique ou de la mort psychique. La culture hospitalière façonne ainsi le travail de care sur cette contrainte-là, qui peut faire office de support pour soutenir le travail psychique individuel mobilisé en cette période de changement à potentialité traumatique, ou bien faire l'effet d'un forçage. Plus précisément, il nous semble que les dispositifs de soins ne proposent pas seulement un encadrement et une scansion, par des propositions rituelles, de ce travail psychique, mais supposent également que le sens est réalisé en lui-même par la pratique du rite. Cette contrainte confère aux ritualités institutionnelles une valeur performative, par laquelle le sujet âgé, initié à l'institutionnalisation des soins au cours d'une hospitalisation, risque de se conformer à ce qu'il perçoit, à ce qu'il constate du fonctionnement institutionnel, et de s'identifier à l'objet même qui focalise l'organisation des soins (Goffman, 1968).

En service de médecine gériatrique, c'est l'ombre du paradigme de la dépendance qui tombe sur l'objet du soin, dont les effets d'assignation ne sont pas à négliger. Ce paradigme de la dépendance est fortement infiltré de constructions sociales négatives entachées des idéaux du " bien vieillir » et par des représentations de rapports sociaux de domination susceptibles d'être intériorisés et exercés par les institutions elles-mêmes. Ce spectre négatif est par ailleurs fortement redoublé par la subordination des financements des institutions aux coûts du soin, et donc par la pression évaluative liée au poids des actes soignants, considérablement alourdi dans les situations de dépendance, évaluation qui reste sous l'emprise d'un modèle biologisant de la dépendance, univers sanitaire oblige. L'assignation d'un vieillissement positivement ou péjorativement connoté, véhiculant l'idée d'un « vieillissement réussi » ou d'un « vieillissement en dépendance », est ainsi susceptible de précéder la perception et d'anticiper la qualification, par le sujet lui-même, des différences dans les modalités du vieillir. Ces messages peuvent dès lors faire courir le risque d'une « assignation 
à dépendance " qui procède d'une véritable prescription (Laplanche, 2007) et alimente un processus identificatoire qui initie finalement le sujet âgé au « métier de vieillard » (Thomas, 2005).

\section{Conclusion}

Ainsi, l'institutionnalisation des soins dont le sujet fait l'expérience inaugurale à l'hôpital peut être appréhendée comme un rite de passage qui non seulement sépare la grande vieillesse des autres âges de la vie, mais qui constitue également un acte d'institution (Bourdieu, 1982), inaugural et fondateur, ayant un effet d'assignation statutaire et de marquage de frontières sociales en projetant le sujet dans la catégorie des " dépendants ». Cet effet d'assignation n'a pas valeur cependant de vérité et ne dit rien de ce que vit subjectivement le sujet âgé, de la façon dont il se laisse faire, s'oppose ou résiste au travail du care, dont dépendent les possibilités de s'approprier le dispositif de soins, voire de le détourner, de le subvertir, de le faire passer du champ de la demande et du besoin vers celui du désir, qui rendent compte de ce que nous appelons l'écho du care. C'est notamment dans cet écart que vient s'inscrire le travail psychique du vieillir, qui ne revient pas à apprendre à être vieux mais à continuer à rêver quelle personne on sera demain, en mettant ses exigences et ses idéaux à l'épreuve du réel de la dépendance, en les remaniant à la mesure de la résistance opposée par un certain nombre de limitations et d'autolimitations éprouvées dans les expériences du corps, endurées dans l'espace du socius.

bibliographie

aulagnier, P. 1975. La violence de l'interprétation. Du pictogramme à l'énoncé, Paris, Puf.

b1eger, J. 1966. « Psychanalyse du cadre psychanalytique », dans R. Kaës (sous la direction de), Crise, rupture et dépassement, Paris, Dunod, 2004, p. 257-276.

boltanski, L. 2008. « Institutions et critique sociale. Une approche pragmatique de la domination », Tracés, $\mathrm{n}^{\circ}$ 8, p. 17-43.

bourdieu, P. 1982. "Les rites comme actes d'institution », Actes de la recherche en sciences sociales, $\mathrm{n}^{\circ} 43$, p. 58-63.

Castoriadis, C. 1975. L'institution imaginaire de la société, Paris, Seuil.

deJours, C. 2016. « Les psychiatres survivront-ils au néolibéralisme ? », Le Carnet psy, $\mathrm{n}^{\circ} 8$, p. 39-42.

frinault, t. 2005. "La dépendance ou la consécration française d'une approche ségrégative du handicap », Politix, n ${ }^{\circ} 4$, p. 11-31.

goffman, e. 1968. Asiles : études sur la condition sociale des malades mentaux et autres reclus, Paris, Éditions de Minuit.

guillemard, A.-M. 2010. Les défis du vieillissement. Âge, emploi, retraite. Perspectives internationales, Paris, Armand Colin. 
kohli, M. 1986. "The World We Forgot: A Historical Review of the Life Course ", dans V.W. Marshall (sous la direction de), Later Life. The Social Psychology of Ageing, Beverly Hills, Sage, p. 271-303.

1aplanChe, J. 2007. «Le genre, le sexe, le sexual », dans Sexual. La sexualité élargie au sens freudien, Paris, Puf, 2014, p. 153-194.

me11ier, D. 2006. "Précarité psychique et dispositifs d'intervention clinique », Pratiques psychologiques, $\mathrm{n}^{\circ} 12$, p. 145-155.

molinier, P. 2010. "Au-delà de la féminité et du maternel, le travail du care », Champ psy, nº 2, p. 161-174.

noë1, J.-L. 2012. « Accompagner le projet de vie en service de soins de longue durée, ehpad, maison de retraite », dans B. Verdon (sous la direction de), Cliniques $d u$ sujet âgé. Pratiques psychologiques, Paris, Armand Colin, p. 235-259.

puget, J. 2005. « Dialogue d'un certain genre avec René Kaës à propos du lien », Le Divan familial, n 2, p. 59-71.

rivière, C. 1995. Les rites profanes, Paris, Puf.

rouchy, J.C. 2006. " La conception du dispositif de groupe dans différents cadres institutionnels ", Revue de psychothérapie psychanalytique de groupe, $\mathrm{n}^{\circ} 47$, p. 9-23.

roussillon, R. 2012. Manuel de pratique clinique, Issy-les-Moulineaux, Elsevier Masson.

thomas, H. 2005. « Le "métier" de vieillard : institutionnalisation de la dépendance et processus de désindividualisation dans la grande vieillesse ", Politix, $\mathrm{n}^{\circ} 4$, p. 33-55.

tronto, J. 1993. Un monde vulnérable. Pour une politique du care, Paris, La Découverte, 2009.

Verdon, B. 2013. Le vieillissement psychique, Paris, Puf. 